\title{
A DiffERENT VIEWPOINT ON PNEUMOCOCCAL GLOMERULONEPHRITIS IN A HEALTHY CHILD
}

Singapore Med J 2014; 55(9): 506 doi: 10.11622/smedj.2014122

\section{Dear Sir,}

I read the case report "Pneumococcal glomerulonephritis in a healthy child: a case report and literature review" by Ismail et al ${ }^{(1)}$ with great interest. However, I have some comments regarding the type of glomerulonephritis (GN) and its causative agent discussed in the paper.

In order to define the type of GN as postinfectious, infectious or others, it is crucial to know the exact time of clinical onset, disease progression and the results of laboratory findings. In Ismail et al's case,(1) renal involvement had started on Day 2 after admission, with acute cervical lymphadenitis. Due to the growth of Streptococcus pneumoniae (S. pneumoniae) in the patient's blood culture, the authors had classified GN as pneumococcal (i.e. infectious GN). They also reported that GN had developed nine days after the initiation of symptoms - seven days before admission and two following hospitalisation - which is an adequate duration of time for the elevation of antistreptolysin $\mathrm{O}$ titre and lowering of complement 3 (C3) level (i.e. postinfectious GN). Despite treatment against $S$. pneumoniae, GN lasting four weeks mandates close monitoring (e.g. biopsy) and immediate interventions to ensure preservation of the kidneys. In fact, it is considered improper to assess the type of GN without first conducting histological testing and monitoring the progress of the $\mathrm{C} 3$ level. This is because clinical findings similar to those reported in this patient may also occur in other histological types of GN.

Furthermore, diarrhoea at onset (an unexpected finding in S. pneumoniae infection), or unresolved fever and progression to bronchopneumonia at Week 2 despite proper antibiotic therapy for two weeks should raise suspicions of the causative agent, and the contribution of different pathogenetical mechanisms should be considered. Therefore, it would be better to include a broad workup in the determination of the causative agent, especially for that of viral or autoimmune origins.

Yours sincerely,

\section{Onur Sakallioglu}

Department of Pediatric Nephrology, Baskent University Faculty of Medicine, Zübeyde Hanım Practicing and Training Hospital, Karşıyaka, Izmir, Turkey. onursakallioglu@hotmail.com

\section{Reference}

1. Ismail IH, Zainudin Z, Othman N. Pneumococcal glomerulonephritis in a healthy child: a case report and literature review. Singapore Med J 2014; 55:e69-72. 